\title{
Improved B-spline Elastic Registration for Medical Image Based on Feature Extraction
}

\author{
Yue Shi, Yangping Wang, Xiaogang Du \\ School of Electronic and Information Engineering, \\ Lanzhou Jiaotong University, \\ Lanzhou, 730070, China \\ E-mail: yue_0667@163.com
}

\author{
Yangping Wang \\ School of Mathematics and Statistics, \\ Lanzhou University, \\ Lanzhou, 730030, China \\ E-mail: w_yp73@163.com
}

\begin{abstract}
In order to improve the accuracy and efficiency of medical image registration, an elastic registration method based on feature extraction and B-spline has been proposed in this paper. The proposed method consists of two steps. First, feature points are obtained by using Harris corner detection algorithm and SIFT algorithm. They are used for the input of Thin Plate Spline method to reduce the scale of deformation by preliminary transformation. It is beneficial to improve the speed of B-spline registration, which is used in the second step of the proposed method. Then the deformation grid is treated as the initial grid of B-spline free-form deformation (FFD), and accurate registration is achieved by $B$-spline transformation of mutual information (MI) driving. Experimental results demonstrate that the running time and registration results of the proposed method are superior to those of B-spline registration due to the combination of the efficiency of preliminary transformation and the accuracy of B-spline.
\end{abstract}

Keywords-medical image; elastic registration; feature extraction; B-spline

\section{INTRODUCTION}

Elastic registration of medical image plays an important role in improving clinical diagnosis, disease surveillance and surgical operation. It uses the nonlinear transformation model to describe complex deformation, such as registration of soft tissue images, different modal images and different individual images. At the current stage, there are two elastic registration methods with one based on feature extraction and the other on image gray ${ }^{[1]}$ : the former has advantages of less calculation, rapid registration and less dependence on image gray, however, its registration accuracy is subject to how accurate the feature extraction is, that is, registration errors would be larger if the feature extraction is not accurate enough; while by contrast, the latter needs no image feature extraction and pretreatment to gain a more accurate registration result, though, it has to take a large amount of calculation and more time. Different methods may be adopted according to different clinical needs.

At present, B-spline free-form deformation (FFD) ${ }^{[2-3]}$ is a common method of elastic registration. Its unlimited degree of freedom and accuracy of local modeling make it the best to describe local deformation. However, it has to depend on the density of the control grid: the deformation would be smooth and less accurate if the control grid is sparse, and vise versa. Although the multi-resolution method helps to resolve the problem, too much running time is still taken due to a lot of iterations.

In order to ensure registration accuracy while increasing the speed of registration, we adopt a combination of registration based on feature extraction and image gray to accomplish complementary advantages. SIFT algorithm ${ }^{[4-5]}$ is too demanding for feature points, while Harris algorithm ${ }^{[6]}$ is a simple and stable feature extraction algorithm, and it extracts more feature points with structure significance. In this paper, Harris algorithm is added in feature extraction process on the basis of SIFT algorithm. Feature extraction is treated as the pretreatment of B-spline registration to achieve registration rapidly and accurately.

\section{Proposed Method}

We use a two-step elastic registration method for medical image. The framework of the method is shown in Fig. 1. The function $\mathrm{R}(X)$ and $\mathrm{F}(Y)$ represent the reference image and the floating image, respectively. $X$ and $Y$ are their definition domains. $\mathrm{S}(t)$ is the similarity function, where $t$ is the control parameter. Mutual information (MI) ${ }^{[7]}$ is treated as similarity measure to evaluate the effect of matching. At this time, registration is to find a deformation function to obtain the maximum of $\mathrm{S}(t)$.

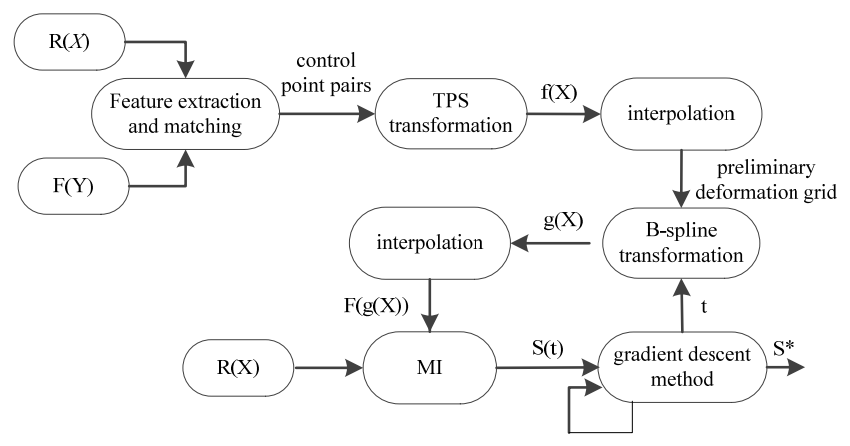

Fig. 1. Framework of the proposed method 
First, the image $\mathrm{R}(X)$ and $\mathrm{F}(Y)$ are inputted. We use Harris corner detection algorithm and SIFT algorithm to obtain feature point pairs. The coordinate of feature points are used for the input of Thin Plate Spline (TPS) method $^{[8-9]}$. TPS transformation is made for $X$ to gain the coordinate of a new region $\mathrm{f}(X)$. Preliminary deformation grid generated by interpolation is treated as the initial grid of B-spline FFD. B-spline transformation is made for $X$ to gain the coordinate of region $\mathrm{g}(X)$. The value of the floating image, $\mathrm{F}(\mathrm{g}(X))$, that is obtained by interpolation. MI is used to calculate the similarity $\mathrm{S}(t)$ between the reference image $\mathrm{R}(X)$ and the interpolated image $\mathrm{F}(\mathrm{g}(X))$. $\mathrm{S}(t)$ is inputted into the optimization module to calculate the optimal transformation parameter $t$ by using the gradient descent method. This process is achieved by iteration until $\mathrm{S}(t)$ gains the maximum. Finally, output the interpolated image $S^{*}$.

\section{A. Feature Extraction and Preliminary Transformation}

Although B-spline FFD can obtain more accurate registration results, it consumes too much running time. We use Harris algorithm and SIFT algorithm to extract feature points of image, and SIFT feature descriptors are generated and matched. The preliminary transformation is treated to the floating image by using TPS method. Depending on the experience, it requires a total of around 200 of feature point pairs to get a better preliminary deformation grid.

SIFT algorithm describes the local feature of the image. It maintains invariance of rotation, scaling and intensity roughness and a degree of stability of the changes of view, affine transformation and noises. SIFT algorithm consists of four steps: scale-space extrema detection, key point localization, orientation assignment and key point description. However, it is too demanding for feature points, therefore, we use Harris corner detection algorithm to increase the number of feature points with structural significance. The basic idea of Harris corner detection algorithm is to move a small displacement in any direction for the image window (generally rectangular region) and calculate the average energy changes of the window. If the energy change value exceeds a given threshold value, the center pixel of window will be regarded as corners ${ }^{[6]}$. The gray of pixel points is denoted as $\mathrm{p}(x, y)$. The energy change of moving $(u, v)$ from each pixel is computed according to:

$$
E_{\mathrm{u}, \mathrm{v}}(x, y)=\sum_{u, v} w_{u, v}(p(x+u, y+v)-p(x, y))^{2}
$$

Where $w_{u, v}$ is a coefficient of gauss window in the position of $(u, v)$. Harris algorithm calculates the autocorrelation matrix for each point of the image and chooses the optimal points using the size of eigenvalues.

After obtaining feature points of two images, the similarity is given to match them by the Euclidean distance between two images' feature vectors. Obtained feature points are used to achieve space transformation by using TPS method. Interpolation is simulated as the bending deformation of a thin metal plate under the point constraint by TPS method ${ }^{[8]}$. Precise fit is reached on the control points of images, and other points are obtained better correction as much as possible under the constraint of control points by interpolation. To make the thin metal plate obtain height $z_{i}$ at the point $\left(x_{i}, y_{i}\right)$ and gain a minimum bending energy, we find a deformation function $\mathrm{f}(x, y)$ in two-dimensional space to get the minimum of penalty function $\mathrm{E}_{s}(f)$. The penalty function can be expressed as:

$$
\left.E_{s}(f)=\iint\left(\frac{\partial^{2} f}{\partial x^{2}}\right)^{2}+2\left(\frac{\partial^{2} f}{\partial x \partial y}\right)^{2}+\left(\frac{\partial^{2} f}{\partial y^{2}}\right)^{2}\right) d x d y
$$

TPS interpolation function is defined as:

$$
f(x, y)=a_{1}+a_{x} x+a_{y} y+\sum_{i=1}^{n} w_{i} U\left(\left|P_{i}-(x, y)\right|\right)
$$

Where $\mathrm{a}_{1}, \mathrm{a}_{x}, \mathrm{a}_{y}$ are the global affine transformation coefficients. $w_{i}$ is the local non-linear transformation coefficients. $U$ is the basis function to structure TPS. $P_{i}$ are the coordinate of $i$ th matching point of the reference image, $i=1,2, \cdots, n .\left|P_{i}-(x, y)\right|$ is the Euclidean distance between control points. The function satisfies $f\left(x_{i}, y_{i}\right)=z_{i}$ for all $i$ and makes the penalty function gain the minimum. The deformation grid is obtained according Eq. (3) and treated as the initial grid of B-spline FFD.

\section{B. Accurate Registration Based on B-spline FFD}

$B$-spline has a great characteristic of local control ${ }^{[10-11]}$. The displacement value of a point in the floating image is only related to its neighboring $4^{2}$ control points. Moving a part of control points is only lead to a limited space around them changing, without making the pixel of entire image to change. Generated deformation grid by TPS is overlaid on the floating image using B-spline FFD. It drives the deformation of the floating image by adjusting the position of control points. The accurate registration is achieved by the above method.

In this paper, cubic B-spline basis function is chosen because of its computing efficiency and second-order continuity ${ }^{[12]}$. Deformation function $g(x, y)$ is constructed in two-dimensional space. The displacement value of each point in the floating image is fitted by that of the control point. The deformation function is expressed as:

$$
g(x, y)=\sum_{l=0}^{3} \sum_{m=0}^{3} B_{l}(u) B_{m}(v) \varphi_{p+l, q+m}
$$

Where $p=\left[\frac{x}{\delta}\right]-1, q=\left[\frac{y}{\delta}\right]-1, u=\frac{x}{\delta}-\left[\frac{x}{\delta}\right], v=\frac{y}{\delta}-\left[\frac{y}{\delta}\right]$ and [ ] is rounding operation. $\mathrm{B}_{l}(l=0, \cdots, 3)$ represents the lth basis function of B-spline. They can be written as:

$\mathrm{B}_{0}(u)=(1-u)^{3} / 6, \quad \mathrm{~B}_{1}(u)=\left(3 u^{3}-6 u^{2}+4\right) / 6, \quad \mathrm{~B}_{2}(u)=(-$ $\left.3 u^{3}+3 u^{2}+3 u+1\right) / 6$,

$\mathrm{B}_{3}(u)=u^{3} / 6$, where $0 \leqslant u<1$. They represent the weight value for control points. They weight the contribution of 
each control point for $\mathrm{g}(x, y)$ by the distance of control points to $(x, y)$.

The generated surface by deformation function $\mathrm{g}(x, y)$ is simultaneously controlled by the $r+3$ control points of $X$ direction and the $s+3$ control points of $Y$ direction. The displacement of points in the floating image is decomposed into $X$ and $Y$ directions and obtained in both directions. It is the parameter to be optimized that the displacement of each control point in the $X$ and $Y$ directions. Each point consists of two parameters to indicate the displacement of the point in the grid. The total number of parameters is $2 \times(r+3) \times(s+3)$, and they are obtained by the optimization process. Each point requires iterative calculation, and the iteration time depends on optimization algorithm and tolerance requirement. Commonly, it adopts a relatively long calculation time to find the optimal parameter settings of transformation.

\section{Algorithm Flow}

Registration for medical image is slower and has a lower accuracy. In this paper, the proposed method solves the problem. It consists of the following four-step:

- Extract feature points of $\mathrm{R}$ and $\mathrm{F}$ by using Harris algorithm and SIFT algorithm. Then SIFT feature descriptors are generated and matched. The coordinate of obtained feature points are denoted as $\left(x_{i}, y_{i}\right)$ and $(x, y)$.

- Obtained feature points are used for the input of the TPS method. Deformation function $\mathrm{f}(x, y)$ is obtained according Eq. (3) and generates preliminary deformation grid.

- The deformation grid is treated as the initial grid of B-spline FFD. Transformation parameters are calculated by combining Eq. (4) and the gradient descent method.

- The similarity function is used to determine whether parameters achieve optimum. If parameters achieve optimum, the optimal solution is obtained. Otherwise, go back and calculate new transformation parameters until MI gains the maximum. Then stop iterating, and registration is completed.

\section{EXPERIMENTS AND RESULTS}

In this experiment, we finished simulation experiments for a large number of clinical images by using the proposed method. The size of images is $512 \times 512$. Experiment platform is Intel (R) Core (TM) i3-2120 CPU (dual core four threads, 3.30GHz), 4G memory. Applications are written in MATLAB V8.0.

Fig. 2 shows the registration results of a group of lung CT images by using B-spline method and the proposed method. The regular grid is used for the initial grid of Bspline FFD, while the deformation grid obtained by TPS transformation is treated as the initial grid of the proposed method to improve the speed of B-spline transformation.

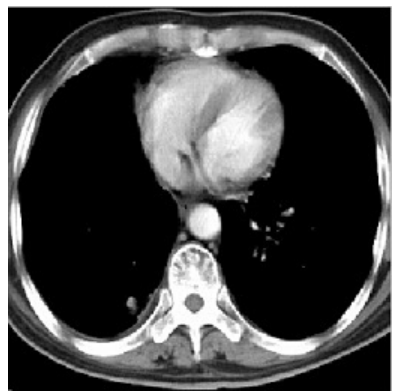

(a)

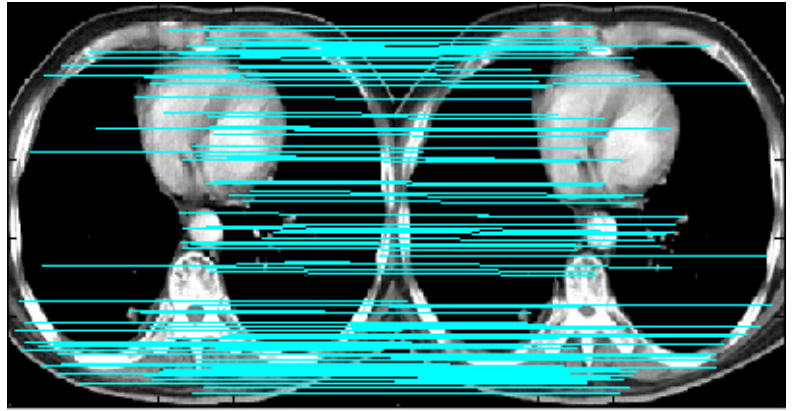

(c)

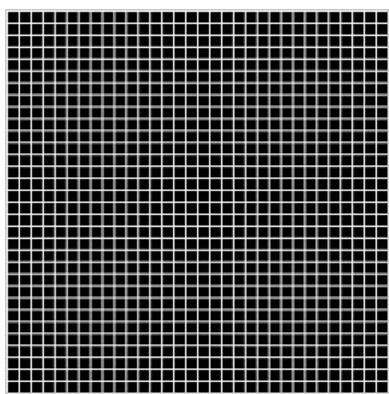

(d)

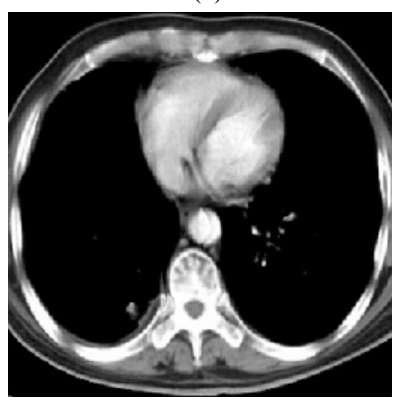

(f)

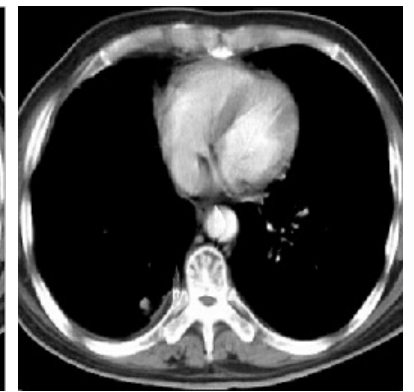

(b)

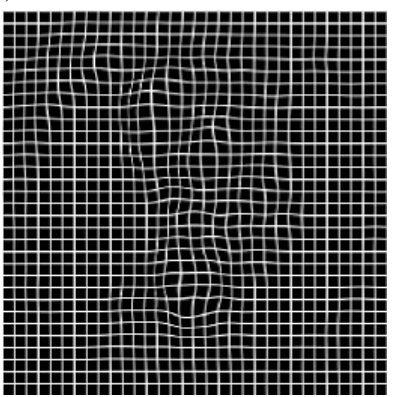

(e)

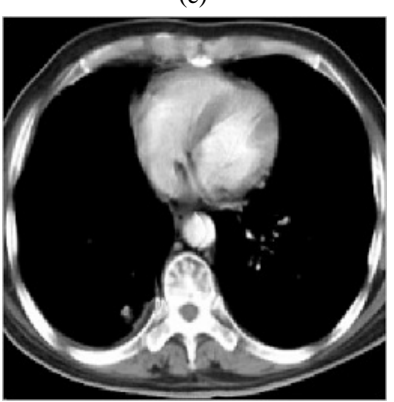

(g)
Fig. 2. Experiment images and registration results. (a) the reference image. (b) the floating image. (c) feature points matching. (d) the initial grid of B-spline FFD. (e) the initial grid of the proposed method. (f) the registration result of B-spline method. (g) the registration result of the proposed method.

The results of registration are evaluated by using $\mathrm{MI}$ and Sum of Squared Differences (SSD). MI is an entropy-based method, and it is one of retrospective registration which has the best registration accuracy and robustness. It can be defined as:

$$
M I(R, F)=H(R)+H(F)-H(R, F)
$$


Where $\mathrm{H}(R)$ and $\mathrm{H}(F)$ are the entropy of $\mathrm{R}$ and $\mathrm{F}$, respectively. $\mathrm{H}(R, F)$ is the joint entropy of $\mathrm{R}$ and $\mathrm{F}$. The larger the value of mutual information is, the higher the similarity of two images has. It represents the registration result is more accurate.

SSD evaluates the effect of registration by the minimum mean square error of two images. It can be written as:

$$
S S D=\frac{1}{N} \sum\left(I_{F}(x, y)-I_{R}(x, y)\right)^{2}
$$

Where $\mathrm{I}_{R}(x, y)$ and $\mathrm{I}_{F}(x, y)$ represent the gray of point $(x$, $y$ ) of R and F, respectively. $\mathrm{N}$ is the total number of pixels in the image region of registration. The larger the value of SSD is, the bigger the difference between two images has. It represents the registration result is worse.

The comparison of registration results between the Bspline method and the proposed method are given in Table I. It can be observed that compared to B-spline method, the proposed method can get a more accurate registration result.

TABLE I. REGISTRATION RESULTS

\begin{tabular}{|l|c|c|c|}
\hline \multirow{2}{*}{ Registration Results } & \multicolumn{3}{|c|}{ Comparison Items } \\
\cline { 2 - 4 } & $\boldsymbol{M I}$ & SSD & $\begin{array}{c}\text { Running } \\
\text { time/s }\end{array}$ \\
\hline before the registration & 1.0137 & 0.0319 & \\
\hline B-spline method & 1.3164 & 0.0053 & 128.51 \\
\hline proposed method & 1.3438 & 0.0041 & 83.53 \\
\hline
\end{tabular}

Fig. 3 shows the comparison of calculation time between the B-spline method and the proposed method. Along the $x$ axis, the gradient tolerance is gradually increasing, which means the decrease of registration accuracy. As can be seen, the B-spline registration takes more calculation time. However, it takes less time to achieve the same accuracy by using the two-step registration method. Moreover, we can observe the advantage of the proposed method is more outstanding when high accuracy is required.

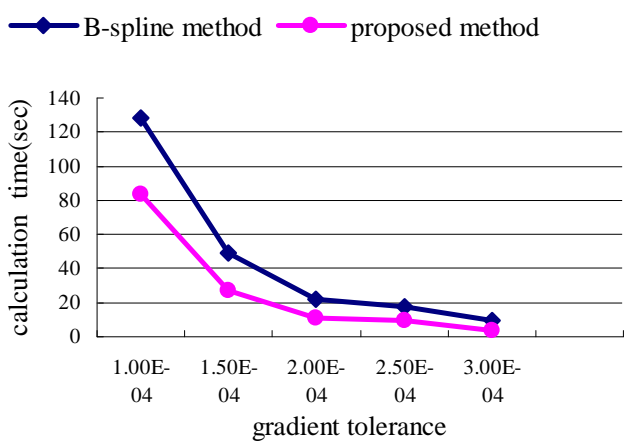

Fig. 3. The comparison of calculation time between the B-spline method and the proposed method.

\section{CONCLUSION}

We have proposed a two-step elastic registration method by combining feature extraction and B-spline. The method improves both running time and registration quality by extracting image features and preliminary transforming the grid of the floating image before B-spline registration. The proposed method improves the registration accuracy significantly when it takes the same time with B-spline method. And it takes less time when they obtain the same accuracy. Through registration and analysis of a large number of clinical images, the results demonstrate that the accuracy and efficiency of the proposed method are improved than B-spline registration.

\section{Acknowledgment}

This work is jointly supported by National Natural Science Foundation (60962004, 61162016), and Gansu International Science and Technology Cooperation Projects (144WCGA162).

\section{References}

[1] A. Sotiras, C. Davatzikos, and N. Paragios, "Deformable medical image registration: a survey,” IEEE Transactions on Medical Imaging, vol. 32, no. 7, pp. 1153-1190, 2013.

[2] D. Rueckert, L.I. Sonoda, C. Hayes, "Nonrigid registration using freeform deformations: application to breast MR images," IEEE Transactions on Medical Imaging, vol. 18, no. 8, pp. 712-721, 1999.

[3] X.H. Zhuang, S. Arridge, D.J. Hawkes, S. Ourselin, "A nonrigid registration framework using spatially encoded mutual information and free-form deformations,” IEEE Transactions on Medical Imaging, vol. 30, no. 10, pp. 1819-1828, 2011.

[4] J. Huo, N. Yang, M.Y. Cao, M. Yang, “A reliable algorithm for image matching based on SIFT,” Journal of Harbin Institute of Technology, vol. 19, no. 4, pp. 90-95, 2012.

[5] H. Goncalves, L. Corte-Real, and J.A. Goncalves, "Automatic image registration through image segmentation and SIFT," IEEE Transactions on Geoscience and Remote Sensing, vol. 49, no. 7, pp. 2589-2600, 2011.

[6] C. Harris and M. Stephens, “A combined corner and edge detector,” Proceedings of the 4th Alvey Vision Conference, Manchester, UK, 1988, pp. 147-151.

[7] S. Klein, M. Staring, and J.P. Pluim, "Evaluation of optimization methods for nonrigid medical image registration using mutual information and B-Splines,” IEEE Transactions on Image Processing, vol. 16, no. 12, pp. 2879-2890, 2007.

[8] F.L. Bookstein, "Principal warps: thin-plate splines and the decomposition of deformations," IEEE Transactions on Pattern Analysis and Machine Intelligence, vol. 11, no. 6, pp. 567-585, 1989.

[9] K Rohr, H.S. Stiehl, R. Sprengel, "Landmark-based elastic registration using approximating thin-plate splines," IEEE Transactions on Medical Imaging, vol. 20, no. 6, pp. 526-534, 2001.

[10] T.J. Jacobson and M.J. Murphy, "Optimized knot placement for Bsplines in deformable image registration," Joint AAPMCOMP Meeting Program, vol. 38, no. 6, pp. 334-338, 2011.

[11] H.Y. Zhang, J.W. Zhang, J.Z. Sun, Y.G. Sun, "Non-rigid image registration algorithm based on B-splines approximation," Transaction of Tianjin University, vol. 13, no. 6, pp. 447-451, 2007.

[12] J.Z. Wang and T.Z. Jiang, "Nonrigid registration of brain MRI using NURBS,” Pattern Recognition Letters, vol. 28, no. 2, pp. 214-223, 2007. 